\title{
EVALUATION OF THE TOTAL AMOUNT OF PHENOL GROUPS IN COMPLEX MIXTURES BY FOLIN'S AND ORAC-FLUORESCEIN METHODOLOGIES
}

\author{
ANA MARIA CAMPOS ${ }^{1}$ CAMILO LOPEZ-ALARCÓN² AND EDUARDO LISSI ${ }^{*}$ \\ ${ }^{I}$ Facultad de Quimica y Biología, Universidad de Santiago de Chile (USACH); \\ ${ }^{2}$ Facultad de Química, Pontificia Universidad Católica de Chile. \\ (Received: July 25, 2012 - Accepted: January 24, 2013)
}

\begin{abstract}
The total number of phenolic HO groups in a mixture and their average reactivity are fundamental parameters in the evaluation of the capacity of the sample to remove potentially damaging free radicals. The total number of phenolic groups is generally determined by Folin's method and several competitive experiments have been proposed to estimate the reactivity of the sample. Among those stand the Oxygen Radical Absorbance Capacity (ORAC) methodologies. However, when fluorescein is employed as target molecule (ORAC-FL methodology) the index is more related to the number of phenolic groups than to their average reactivity. In the present work it is shown that both indexes correlate with the number of phenolic groups in pure compounds and with the total number of reactive OH groups in complex mixtures (beverages, infusions, fruits and vegetables). This leads to a fair correlation between Folin's and ORAC-FL indexes in these samples.
\end{abstract}

Keywords: Phenols; Folin's method; ORAC-Fluorescein; antioxidants.

\section{INTRODUCTION}

The amount of phenol groups present in an infusion ${ }^{1}$, beverage ${ }^{2}$, juice, fruit $^{3}$ or vegetable ${ }^{4}$ is a relevant parameter, mainly due to their radical trapping capacity ${ }^{5}$. There are a large number of methodologies aimed to the evaluation of the total antioxidant capacity of these mixtures ${ }^{6}$. However, different procedures, and sometimes the same technique employing different target molecules, render different results ${ }^{7}$. This is due to the fact that procedures evaluating total antioxidant capacities provide indexes that are determined by the amount of antioxidants ${ }^{8}$, their reactivity ${ }^{9}$, and the secondary reactions of the radicals derived from the additives and/or the target molecule ${ }^{10,11}$. Comparison of different indexes can provide information regarding the amount and/or reactivity of the phenolic $\mathrm{HO}$ groups present in the considered mixture ${ }^{1}$. In order to have an estimate of the quality of the antioxidants present in a sample in which it is tested its antioxidant capacity, it is important to have an independent evaluation of the amount of phenol groups present in the sample. This evaluation is generally carried out by titration of phenolic moieties with Folin's reactant ${ }^{11-14}$. This is a robust test since gallic acid added to wine is recovered quantitatively ${ }^{15}$, and the absorbance produced by a mixture of natural phenols of different classes is equivalent to the sum of their individual contributions ${ }^{16}$. Unfortunately, there are very few works aimed to establish the capacity of this procedure to titrate all phenol groups present in a polyphenol or in a complex mixture of compounds. In particular, there are few studies devoted to establish the relationship between the number of phenolic HO groups in a compound and its response to the Folin's assay. Ma and Cheung related the differences observed to changes in the absorption coefficient of the phosphotungstates-phosphomolybdates complexes ${ }^{12}$, concluding that substituted methoxyl groups could improve the response of a phenol group towards Folin's reactant. Furthermore, they state that the degree of hydroxylation correlates well with the molar absorbance determined by Folin's method. However, Singleton et al..$^{17}$ concluded that not all phenolic HO groups are titrable and that extra phenolic groups can be generated during the sample treatment. Furthermore, the molar absorptivity per reactive group could depend upon the phenol structure, changing by nearly a two factor.

In the present work we compare the Folin's index obtained for a large number of compounds bearing from one to six phenolic HO groups and test the possibility that evaluation of the ORAC index employing fluorescein as target molecule could provide an alternative (or complementary) methodology to estimate the amount of reactive centers in polyphenols and their complex mixtures. In previous publications, we have shown that protection of fluorescein damage by peroxyl radicals is characterized by clear induction times ${ }^{7-9}$. An open question is whether lag phase measurements directly reflects the quantity of reactive phenolic groups ${ }^{18,19}$. This type of correlation has been reported for flavones and flavanones employing $\beta-\mathrm{PE}$ as target molecule, compound prone to give clear induction times in ORAC methodologies ${ }^{13}$. Furthermore, it is remarkable that, even in complex mixtures, there are a fair correlations between total phenols (evaluated by Folin's) and ORAC values obtained employing fluorescein (ORAC-FL) as target molecule ${ }^{1,20,21}$.

\section{EXPERIMENTAL}

\subsection{Evaluation of total phenolic HO groups by Folin's method.}

Total phenols content in pure compounds and their complex mixtures were determined by the Folin-Ciocalteau colorimetric method ${ }^{1}$ Brieflyl, appropriate dilutions of the samples were added to $0.2 \mathrm{~N}$ Folin-Ciocalteau reagent (Merck Darmstadt, Germany) and, after 5 minutes, sodium carbonate $(75 \mathrm{~g} / \mathrm{L})$ was added. The reaction mixture was incubated for two hours at room temperature and the absorbance of the resulting blue coloured complex was measured at 740 $\mathrm{nm}$. Measurements were carried out in a UV-160 Shimatzu spectrophotometer. Trolox was employed as standard phenolic compound and the results expressed in $\mathrm{mM}$ equivalents.

\subsection{Evaluation of antioxidants by ORAC-Fluorescein method.}

The consumption of fluorescein resulting from its incubation in presence of 2,2'-azo-bis(2-amidinopropane)dihydrochloride (AAPH) was estimated from fluorescence $(\mathrm{F})$ measurements. ${ }^{1}$ Values of $\mathrm{F} / \mathrm{F}^{\circ}$ were plotted as a function of time and the area under the curve (AUC) was obtained by graphical integration. Comparison of the area under the curve for the sample and that of a standard Trolox solution allows an estimation of the ORAC of the sample in Trolox mili equivalents according to

$\left.\mathrm{ORAC}=\left\{\left(\mathrm{AUC}_{\mathrm{AUC}}\right)^{\circ}\right) /\left(\mathrm{AUC}_{\mathrm{Trolox}}-\mathrm{AUC}^{\circ}\right)\right\}($ Trolox $) /($ Additive $)$

for the pure compounds, and

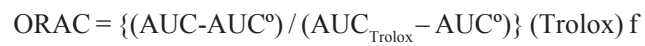

Were $\mathrm{f}$ is the dilution factor, for the complex mixtures.

\section{RESULTS AND DISCUSSION}

3.1 Pure Compounds index.

3.1.1. Number of phenolic HO groups in the molecule and Folin's

Folin values obtained for different phenols are compiled in Figure 1. 


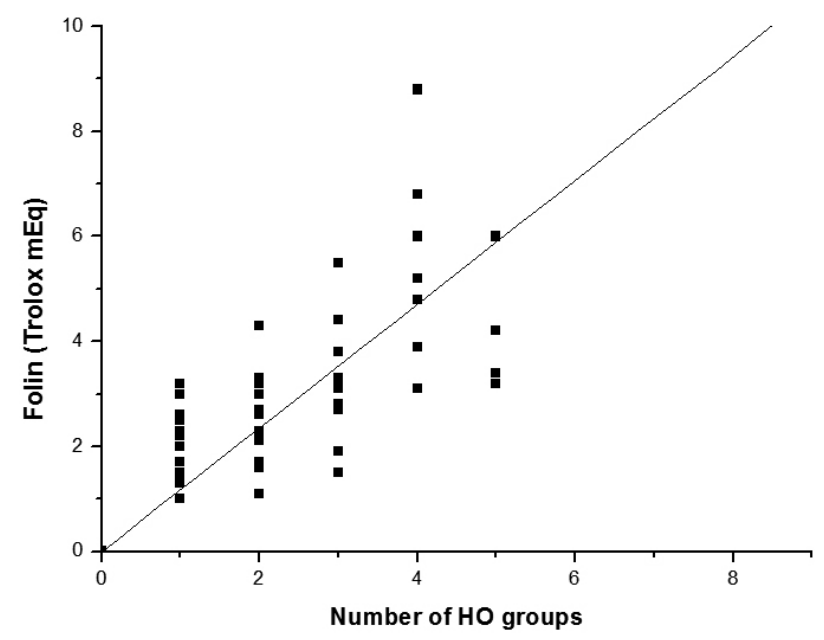

Figure 1. Folin's values for pure compounds, expressed in Trolox equivalents, plotted as a function of the number of phenolyc $\mathrm{OH}$ groups. Data reported in Refs. 4, 23, 14, 25, and in the present work.

These data have been collected from different sources, a fact that could contribute to the observed dispersion of the values. When more than a value has been reported the average value is considered. These data show that a significant correlation is observed between the number of phenolic HO groups in the molecule, and the absorbance of the complex produced in the Folin's methodology. The statistics of the correlation is given in Table 1 .

Table 1. Summary of the statistical relationship between number of phenolic HO groups in pure compounds, Folin's index and ORAC-FL values (expressed in Trolox equivalents).

\begin{tabular}{|c|c|c|c|c|}
\hline Correlation & $\mathrm{N}^{\mathrm{a}}$ & $\mathrm{B}^{\mathrm{b}}$ & $\mathrm{R}^{\mathrm{c}}$ & $\mathrm{p}^{\mathrm{d}}$ \\
\hline Folin index vs $\mathrm{N}^{\mathrm{o}}$ of HO & 58 & 1.18 & 0.73 & $<0.0001$ \\
\hline ORAC-FL vs N $\mathrm{N}^{\mathrm{o}}$ of HO & 24 & 1.36 & 0.62 & 0.001 \\
\hline ORAC-FL vs Folin index & 18 & 1.5 & 0.56 & 0.014 \\
\hline
\end{tabular}

(a) Number of compounds included

(b) Slope of the linear relationship

(c) Correlation coefficient

(d) Correlation probability

The average value of the Folin Index/number of phenolic HO groups is 1.18 , with values ranging from 0.5 (naringine) up to 2.2 (ellagic acid).

\subsubsection{Number of phenolic HO groups in the molecule and ORAC-FL} values.

Figure 2 shows ORAC-FL indexes for a variety of phenols and polyphenols plotted as a function of the number of reactive centres in the corresponding compound .

The data given in figure show a moderate correlation $(\mathrm{R}=0.62)$ with a positive slope $(B=1.36)$ and a significant $p$ value $(p=0.001)$. The range of values of the ORAC-Fl ratio is from 0.33 (gallic acid) to 3.6 (apigenin). The results are summarized in Table 1 . These results would indicate that the ORAC-FL value of a given compound is mostly determined by the number of reactive groups and the stoichiometry of the process. The low values given for some compounds, in particular those bearing a large number of potentially reactive groups, can be due to the presence of unreactive groups and/or to the occurrence of short chains when the primary radicals are prone to give quinones by reactions of the semi-quinone radicals with oxygen.

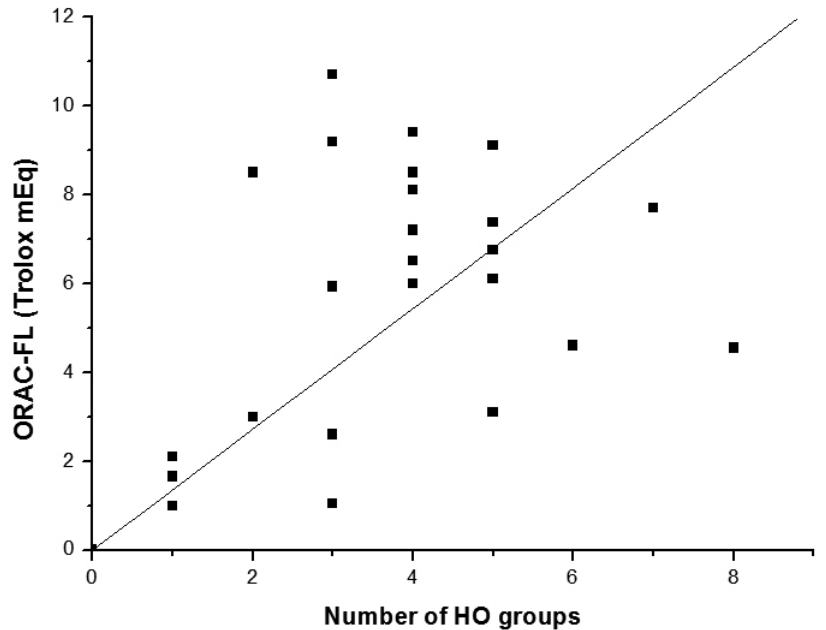

Figure 2. ORAC-FL values for pure compounds, expressed in Trolox equivalents, plotted as a function of the numbers of phenolyc $\mathrm{OH}$ groups. Data reported in Refs.24, 25, 26, and obtained in the present work.

\subsubsection{Correlation between ORAC-FL and F-C indexes.}

The correlation between ORAC-FL values and Folin's index for individual compounds is shown in Figure 3 and the derived statistical parameters included in Table 1.

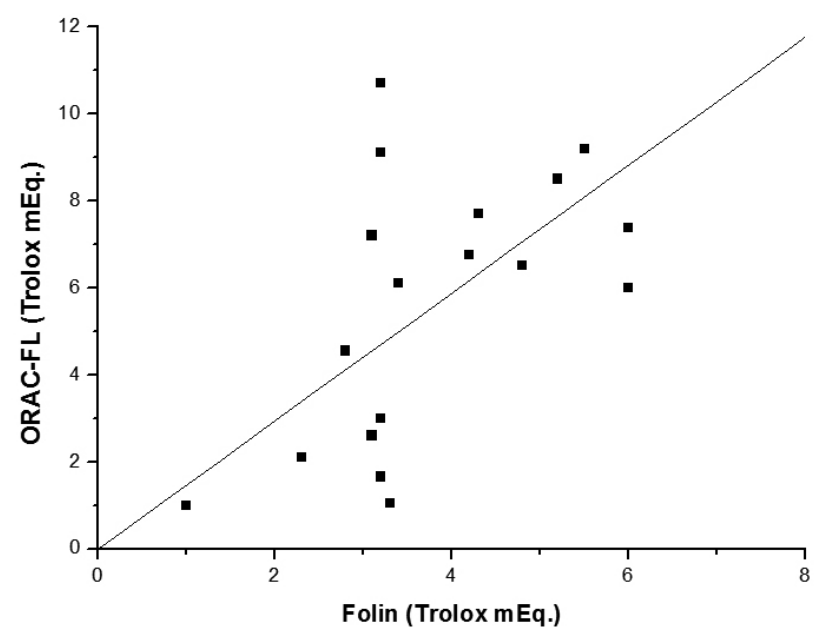

Figure 3. ORAC-FL values for pure compounds plotted as a function of the Folin's index of the corresponding compound.

These data show a fair correlationship between both indexes and the average value of the ORAC-F1/Folin index ratio is ca. 1.5. The actual values of this ratio range from. 0.3 (gallic acid) to 3.3 (apigenin). The positive correlation between both indexes values confirms the relevance of stoichiometric factors on ORAC-FL values. A remarkable aspect of the data collected in Table 1 is the fact the slopes of the plots, represented by the parameter B, are rather similar and close to one. This again emphasizes the dominant role of stoichiometric factors in the ORAC-FL assay.

However, it is interesting to note that the correlation between both indexes is smaller than those derived from the plots shown in Figs. 1 and 2. This would indicate that compounds with particularly large (or small) values of ORAC-FL not necessarily present similar peculiar values for the Folin index.

The data of Table 1 indicate that the best correlation with the number of phenolic HO groups is that of Folin's values, suggesting other factors, such as the compound reactivity and/or the secondary reactions of the phenol, contributes to the ORAC-Fl value. 


\subsection{Complex mixtures}

In complex mixtures comprising different phenolic HO groups bearing compounds it could be expected a positive correlation between ORAC-Fl and Folin's indexes. Furthermore the slope of ORAC-FL vs Folin plots must lay between 0.33 to 3.3 , values that cover all the range for the single compounds. This type of plot is shown in Figure 4 for a series of herbal infusion, including teas ${ }^{1}$, and Chilean wines ${ }^{9}$.

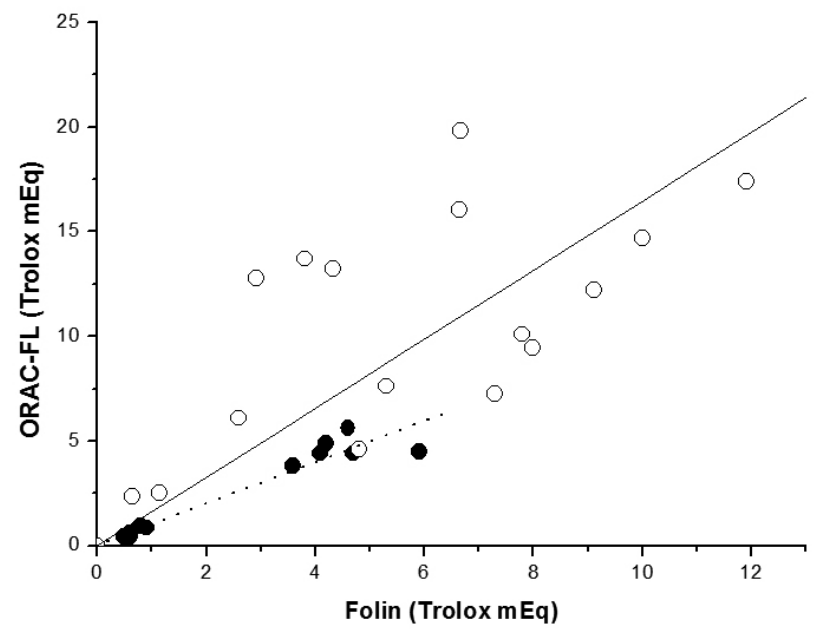

Figure 4. ORAC-FL values for wines ${ }^{9}(\bullet)$ and teas and infusions ${ }^{1}(\mathrm{o})$ plotted as a function of the corresponding Folin's index.

The data collected in this figure clearly support the first premise since both plots shows a strong positive relationship between ORAC-FL indexes and Folin's values. Furthermore, as expected, the slope B measured for teas and herbal infusions is 1.64 , value very close to that reported for individual compounds (see Table 1). Similar results have been reported for Chilean wines, extracts from fifteen selected plant species from the Amazonian region, vegetable extracts, and eight Spanish wines. These data are summarized in Table 2 .

Table 2. Relationship between ORAC-FL and Folin's indexes reported for different antioxidant mixtures, expressed in Trolox equivalents.

\begin{tabular}{|c|c|c|c|}
\hline Sample & ORAC/Folin & $\mathrm{R}$ & Ref \\
\hline Individual compounds & 1.5 & 0.56 & This work \\
\hline Teas and herbal infusions & 1.64 & 0.76 & 1,27 \\
\hline Chilean Wines & 1.1 & 0.97 & 9 \\
\hline Spanish Wines & 1.44 & 0.99 & 23 \\
\hline Amazonian plants & 1.31 & 0.83 & 20 \\
\hline Vegetable extracts & 0.96 & 0.95 & 22 \\
\hline
\end{tabular}

This table shows that the ratios are close to one, well inside the predicted range. Differences in these ratios can reflect the contributions of phenols with high or low ORAC responses. Several factors can contribute to these differences:

i) Crowding of phenolic $\mathrm{OH}$ groups, such as in gallic acid derivatives, can decrease the ORAC-Fl / Folin ratio. This is stressed by the fact that gallic acid, in spite of its three potentially reactive groups, has an ORAC-FL index similar to that of Trolox,

ii) Low reactivity of peroxyl radicals towards the antioxidants could reduce the ORAC-FL index;

iii) Secondary reactions of the phenol derived radicals that take place with high rates and repair the FL derived radicals, such as those leading to quinonelike products, can increase the ORAC-Fl index; iv) The oxidation potential of the antioxidant determines the shape of the $\mathrm{Fl}$ consumption lag phase and, hence, the ORAC value. Bisby et al. have shown that the lag phase becomes less marked with increasing oxidation potential of the antioxidant, rendering low ORAC-FL values ${ }^{11}$.

The contribution of all these factors would explain the rather low correlation between ORAC-FL and Folin indexes observed in Figures 2 and 3 and Table 3. Furthermore, Fig. 1 and Table 3 show that some dispersion of the data takes place also for the Folin index, although the correlation with the number of phenolic HO groups is better than that derived from ORAC-FL values.

A remarkable conclusion from this Table is that the correlation between ORAC and Folin's indexes is considerably better in complex mixtures than when individual antioxidants are considered. This is an expected result if it is considered that any complex sample comprises several antioxidants combining compounds of high and low ORAC responses, making unlikely extreme values, as observed when individual compounds are considered.

\section{CONCLUSIONS}

In pure compounds, both Folin's and ORAC-FL indexes correlate with the number of phenolic $\mathrm{HO}$ groups in the molecule considered. This correlation also holds for complex mixtures, where there exists a fear correlation between the values of both indexes. This implies that ORAC-FL values are mostly determined by the total number of phenolic $\mathrm{HO}$ groups in the sample.

\section{ACKNOWLEDGMENT} Chile)

This work has been financed by DICYT (Universidad de Santiago de

\section{REFERENCES}

1. A. Poblete C. Lopez-Alarcón, E. Lissi, and A. M. Campos. J. Chil. Chem. Soc. 54 (2009) 154-157.

2. C. Lopez-Alarcón and E. A. Lissi. Free Radical Res. 40 (2006) 979.

3. R. H. Liu, American J. Clin. Nutrition. 78 (2003) 5175-5205.

4. P. Stratil, B. Klejdus, and V. Kuban. J. Agric. Food. Chem. 54 (2006) 607-616.

5. A. C. Rice-Evans, N.J. Miller and G. Paganga. Free Rad. Biol. Med. 20 (1996) 933-956.

6. V. Rojinsky and E. Lissi. Review of methods to determine chain breaking antioxidant activity in food. Food Chem. 92 (2005) 235-254.

7. C. Lopez-Alarcón, A. Aspée and E. Lissi. Influence of the Target molecule on the ORAC index. In Emerging Trends in Dietary Components for Preventing and Combating Disease. Editor(s): Bhimanagouda S. Patil, Guddadarangavvanahally K. Jayaprakasha, Kotamballi N. Chidambara Murthy, and Navindra P. Seeram. ACS Bokds, Volume 1093 (2012) Chapter 24, 417-429.

8. C. Lopez-Alarcón and E. Lissi. Free Radic. Res. 39 (2005) 729-736.

9. C. Lopez-Alarcón, R. Ortiz, J. Benavides, E. Mura and E. Lissi. J. Chil. Chem. Soc., 56 (2011) 682-685.

10. C. Lopez-Alarcón, A. Aspee and E. Lissi. Free Radic. Res. 41 (2007) 1189-1194.

11. R. H. Bisby, R. Brooke, and S. Navaratnam. Food Chem. 108 (2008) $1003-$ 1007.

12. Y-T Ma and P.C. Cheung. J. Agric. Food Chem. 55 (2007) 4222-4228.

13. C. Cao, E. Sofic and R. L. Prior. Free Rad. Biol. Med. 22 (1997) 749-760.

14. N. Nenadis, S. Boyle, E. C. G Bakalbassis and M. Tsimidou JAOCS 80 (2003) 451-458.

15. V.L. Singleton and J.A. Rossi, Am. J. Enol. Vitic. 16 (1965) 144.

16. V.L. Singleton, Adv. Chem. Ser. 134 (1974) 184.

17. V.L. Singleton, R. Orthofer, and R. M. Lamuela-Raventós. Methods in Enzimology, 299 (1999) 152-178.

18. E. Niki, Nutrition 18 (2002) 524-525.

19. E. Niki and N. Noguchi, J. Agric. Food Chem. 55 (2007) 323-329.

20. E. M. Silva, J. N. Souza, H. Rogez, J. F. Rees and Y. Larondelle, Food Chem. 101 (2007) 1012-1018.

21. R. N. Prior, X. Wu and K. Schiaichi. J. Agric. Food Chemistry 53 (2005) 4290-4302.

22. M. Ciz, H. Cisová, P. Deneb, M. Kratchanova, A. Slavov, A. Lojek. Food Control 21 (2010) 518-523. 
23. A. Dávalos, C. Gómez-Cordovés, B. Bartolomé. J. Agric. Food Chem. 52 (2004) 48-54.

24. K. L. Wolfe and R. H. Liu. J. Agric. Food Chem. 56 (2008) 8404-8411.

25. N. Nenadis, O. Lazaridou and M. Z. Tsimidou. J. Agric. Food Chem. 55 (2007) 5452-5460.
26. B. Ou, M. Hampsch-Woodill and R. Prior. J. Agric. Food Chem. 49 (2001) 4619-4626.

27. E. Alarcón, A.M. Campos, A.M. Edwards, E. Lissi and C. López-Alarcón. Food Chem, 107 (2008) 1114-1119. 